\title{
Perineostomy: the last oportunity
}

\author{
Juan Carlos Regueiro Lopez ${ }^{1}$, Enrique Gomez Gomez ${ }^{1}$, Alberto Alonso Carrillo ${ }^{1}$, Roque Cano Castiñeira ${ }^{1}$, \\ Maria Jose Requena Tapia ${ }^{1}$
}

${ }^{1}$ Department of Urology, Reina Sofía Universitary Hospital, Córdoba, Spain

\begin{abstract}
Objective: To review the technique and outcome of perineal urethrostomy or urethral perineostomy and to identify factors related to the procedure failure.

Material and methods: We studied 17 patients who underwent perineal urethrostomy between 2009-2013 in a single hospital. Success was defined as no need for additional surgical treatment or urethral dilatation. We reviewed the clinical data related to age, weight, previous urethral surgery, diabetes, hypertension, ischemic cardiopathy, lichen sclerosus and other causes and studied their association with the procedure failure (univariate analysis). We completed the analysis with a multivariate test based on binary regression.

Results: The average follow-up was 39.41 months. From all the causes, we found Lichen Sclerosus in 35\%, idiopathic etiology in 29\% and prior hypospadia repair in 18\%. Postoperative failure occurred in 3 patients, with a final success of $82.4 \%$. The binary regression model showed as independent risk factors ischemic cardiopathy (OR: 2.34), and the presence of Lichen Sclerosis (OR: 3.21).

Conclusions: The success rate with the perineal urethrostomy technique shows it to be a valid option above all when we preserve the urethral blood supply and plate. Lichen sclerosus and ischemic vascular problems are risk factors to re-stenosis.
\end{abstract}

\section{ARTICLE INFO}

\author{
Key words: \\ Perineum; Lichen Sclerosus et \\ Atrophicus; Balanitis Xerotica \\ Obliterans
}

Int Braz J Urol. 2015; 41: 91-100

Submitted for publication: October 29, 2013

Accepted after revision: June 01, 2014

\section{INTRODUCTION}

There are numerous methods for the treatment of anterior urethral stricture, from simple dilatation, to endoscopic procedures and urethroplasties, with variable success rates but often very high. However, when the stenosis affects the anterior urethra, finding a suitable process becomes a challenge. Pan-urethral stenosis can have multiple causes, but when the etiology is lichen sclerosus (LS) or balanitis xerotica obliterans (BXO) the use of multiple grafts forces us to look for non-skin graft sources $(1,2)$. Factors associated with failure in the reconstruction are previous hypospadias surgery, urethroplasty and pelvic radiation due to oncological processes (3-5).
If the anterior urethra is not suitable for successful reconstructive procedures because of severe, complex and refractory stenosis, we can consider the perineal urethra (perineal urethrostomy or perineostomy) which lies proximal to the stenosis and distal to the striated sphincter, not only to prevent bladder damage but also renal. Some patients reject a second or third urethral stage and decide to keep perineal micturition. This technique is widely accepted as the first step in a sequential reconstruction process or as the only step if an adequate reconstruction is not possible (6-9). This technique is always offered to older patients or patients who would not undergo long reconstructive urethroplasty procedures, or as a single solution to the presence of a LS. 
In our study we describe the use of perineal urethrostomy in a varied group of patients in which LS is not the only cause of the pan-urethral stenosis. Importance is given in the technique to maintain the urethral plate and thus the entire urethra vascularization, as do other authors (10). The concept of urethra marsupialization was described in 1914. Its application to the Leadbetter perineostomy was initiated in 1960, and improved by Johanson and Blandy following the principles of Turner-Warwick $(7,11)$. In 1968, the use of the inverted U-scrotal flap was defined (7). The scrotal flap is difficult to achieve in patients who are very obese or have altered perineal anatomy, therefore other forms of skin pedicle grafts are performed, in order to avoid the risk of complications as high as 30\% in some series (6).

This study details the surgical technique and reviews in our cohort the clinical performance of perineal urethrostomy, and it evaluates statistically the variables associated with the success of the procedure.

\section{MATERIALS AND METHODS}

A retrospective study of all perineal urethrostomies carried out and followed-up between 2009-2013 in a single center was performed. We reviewed different clinical variables. Success was defined as no need for additional surgical treatment or urethral dilatation.

We conducted a univariate and multivariate analysis to determine factors associated with the risk of failure, using chi-square and ANOVA tests for qualitative and quantitative variables respectively, and a logistic regression was used to the multivariate analysis. Analyses were performed using SPSS Statistics software version 17.0.

\section{SURGERY TECHNIQUE}

The patient is usually placed in a high lithotomy position and loco-regional anesthesia is applied. An inverted U-shaped incision is made, placing the tip of the $U$ in the upper portion of the perineum where the scrotum ends in order to create a complete non-tense pedicle, which includes fat, on the urethral plate. The bulboca- vernosus muscle is separated to expose the bulbar urethra, which is sectioned 4-6 cm longitudinally, and then hemostatic sutures are employed on the edges (Figure-1).

Figure 1 - Urethrotomy and hemostatic sutures.

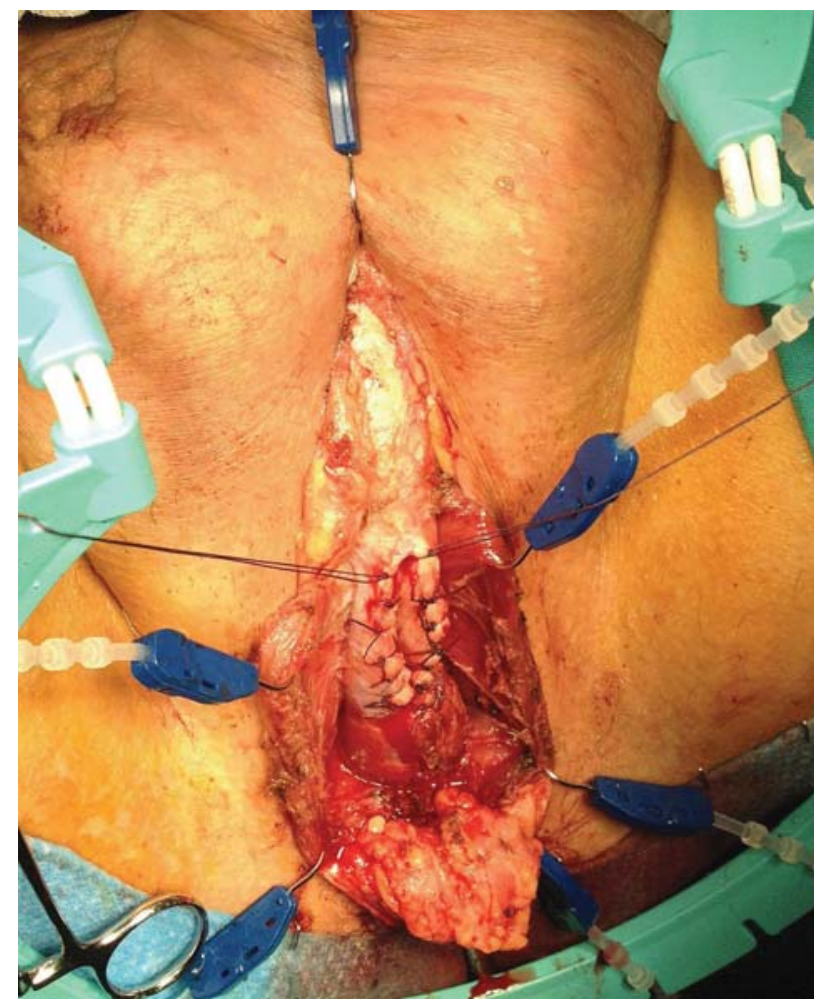

Then we proceed with the urethral calibration up to the bladder to avoid unnoticed deeper stenosis. The ventral incision can be extended to membranous urethra free of stricture. Perineum skin is sutured to the edges of the ventral urethrotomy, preserving the urethral dorsal plate. The apex of the $U$ is sutured to the proximal edge of the urethrotomy, using an absorbable suture polyglactin (Vicryl) 3-0 or 4-0. Once the process of suturing the skin edges to the urethrotomy is complete, there are two incisions remaining, which are then sutured (Figure-2). The complete urethrostomy has two entrances, one proximal leading to the bladder and the other distal leading to the penis.

The urethra is not sectioned in its entirety, only longitudinally, maintaining blood flow 
in the corpus spongiosum. A Foley $16 \mathrm{~F}$ silicon catheter is inserted.

Figure 2 - Post-surgical appearance.

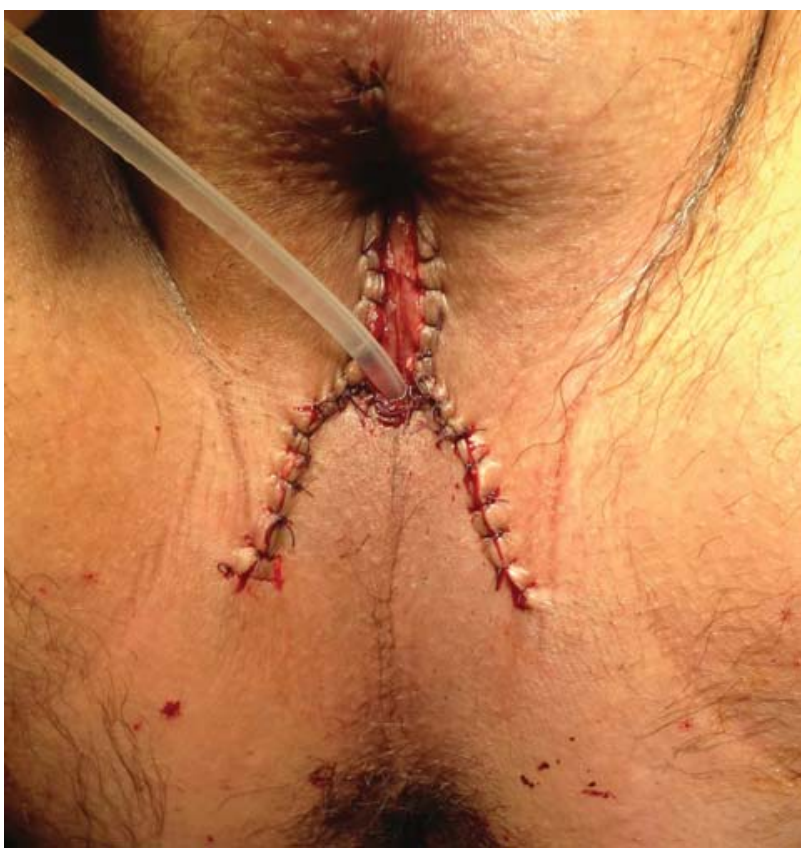

\section{Postoperative course}

The patient ambulates on the afternoon of the operative day. The catheter is left in place for 10 days to promote complete healing of the suture. Patients are discharged from the hospital the day after surgery.

Uroflowmetry and urine culture are repeated every 4 months in the first year and annually thereafter.

\section{RESULTS}

From 2009-2013, 17 patients with a mean age of 65.88 years (range 50-92) underwent perineal urethrostomy. The etiology of pan-urethral stenosis was multifactorial in many cases, idiopathic in 5 (29\%), LS or BXO in 6 (35\%), prior hypospadias repair in $3(18 \%)$, and one case of penile carcinoma, one of radiotherapy, and one iatrogenic case. In many cases, repeated episodes of infection overlap contributed to increased urethral stricture.
In all patients, pan-urethral stenosis was defined as a stenosis which affected the whole anterior urethra (Figures 3 and 4). Five patients (30\%) presented suprapubic catheterization. Previous surgery of urethroplasty was found in 6 patients (35.3\%), and internal urethrotomy in 8 (47\%), with $87.5 \%$ of these having undergone two previous internal procedures.

Among the medical history of interest, chronic ischemic heart disease was observed in 6 patients (35\%), diabetes mellitus in $6(35 \%)$ and hypertension in 10 medicated patients (59\%). The average weight was estimated at $79.82 \mathrm{~kg}$ (65106), with an age of over 60 in 10 patients.

The mean follow-up was 39.41 months (3 months-91 months), with all patients receiving complete follow-up. Immediate complications were related to perineal wound bleeding in one case, and maintenance of urinary catheter for longer time due to delayed perineal wound in three cases.

Postoperative stenosis or failure occurred in 3 patients with a final success rate of $82.4 \%$. All patients undergo calibration tracking. The mean time to failure diagnosis was 4 months (2-6 months).

We performed a univariate analysis to identify causes related to failure of the procedure and development of new stenosis. The results are shown in Table-1.

When a binary regression study was carried out the presence of ischemic heart disease (OR 2.34, 95\% CI [1.08 to 4.98]) and the presence of LS (OR 3.21, 95\% CI [1.00 to 4.89]) were shown as independent factors associated with procedure failure. Results are shown in Table- 2 .

\section{DISCUSSION}

The concept of complex anterior urethral stricture includes pan-urethral stenosis affecting bulbar and penile urethra simultaneously and patients with urethral strictures who have previously undergone failed urethroplasty. The LS is the most common cause of pan-urethral stricture (12), and failed hypospadias correction is the major cause of anterior complex stricture $(13,14)$. It remains difficult and controversial to recom- 
Figure 3 - Panurethral stenosis. Retrograde urethrography.
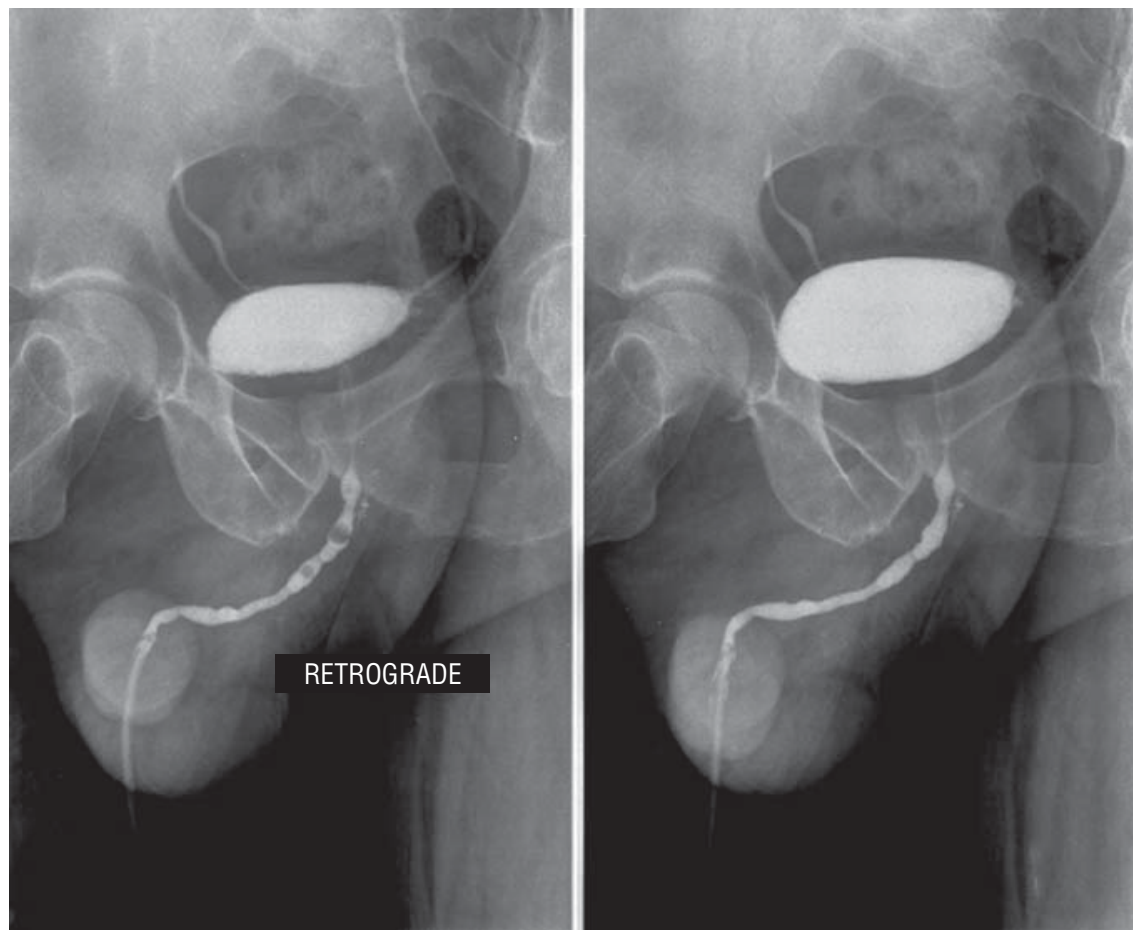

Figure 4 - Panurethral stenosis. Voiding urethrography.

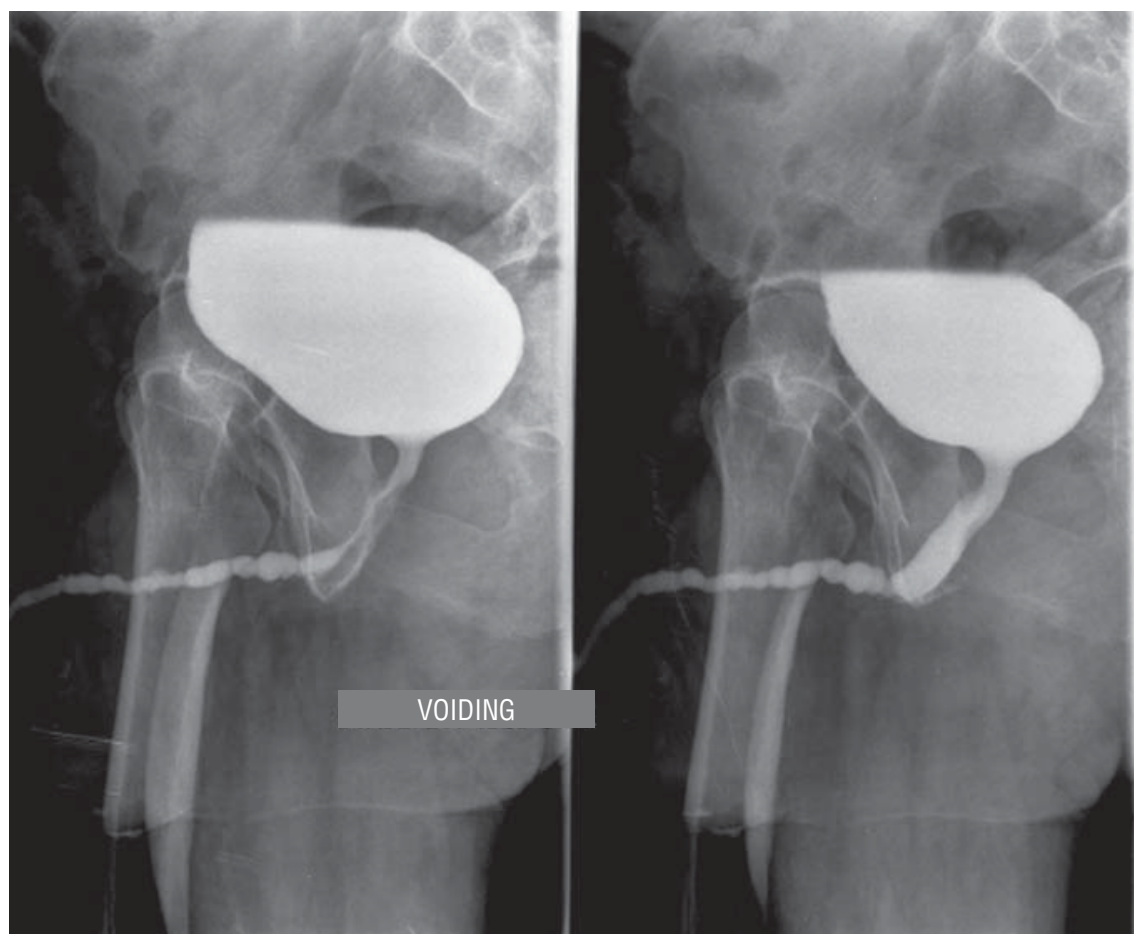


Table 1 - Variables related to procedure success.

\begin{tabular}{lccc}
\hline Qualitative Variables & RR & $95.0 \% \mathrm{Cl}$ & P-Value* $^{*}$ \\
\hline Ischemic heart disease & 4.67 & $1.71-12.72$ & 0.01 \\
Diabetes & 2.33 & $0.74-7.38$ & 0.20 \\
Hypertension & 2.00 & $0.97-3.38$ & 0.10 \\
Previous urethroplasty & 1.75 & $0.94-2.75$ & 0.15 \\
Previous Urethrotomy & 2.33 & $0.98-6.56$ & 0.07 \\
Previous Radiotherapy & 1.16 & $0.94-1.44$ & 0.48 \\
Previous Hypospadia repair & 1.26 & $0.96-1.67$ & 0.37 \\
Penile Cancer surgery & 1.27 & $0.97-1.67$ & 0.37 \\
Lichen sclerosus & 3.50 & $1.52-8.01$ & 0.02 \\
Quantitative variables & Group success average & Failure Group average & p-value \\
Age & 62.57 & 81.33 & 0.02 \\
Weight & 81.57 & 71.67 & 1.14 \\
Number of previous Urethrotomies & 0 & 0.13 \\
\hline
\end{tabular}

*Statistical significance based on univariate analysis and Student's $\mathrm{t}$ - test.

Table 2 - Odds Ratio (Binary regression).

\begin{tabular}{lcccc}
\hline Variables & P value* $^{*}$ & $\mathrm{RR}$ & \multicolumn{2}{c}{$95.0 \% \mathrm{Cl}$} \\
\hline IHD & & & Inferior & Superior \\
\cline { 2 - 4 } BXO & 0.03 & 2.32 & 1.08 & 4.99 \\
\hline
\end{tabular}

IHD = Ischemic heart disease; $\mathbf{B X O}=$ Balanitis xerotica obliterans

*Statistical significance based on multivariate analysis.

mend a particular technique in these cases (15). Most studies of perineal urethrostomy have LS as the underlying disease. The LS is a chronic dermatological autoimmune inflammation that causes discomfort and morbidity that involves both urinary and sexual problems (itching, pain, phimosis and weak stream). The true incidence and prevalence is unknown. The age of onset is between the ages of 40 and 50, although it has been reported in adolescents and children. Its name has generally been LS, but urologic literature has referred to it as BXO (balanitis xerotica obliterans). Its exact etiologic risk factors remain unknown; suggesting an autoimmune dysregulation, viral or bacterial infection, local trauma, chemical irritation or hormonal dysfunctions. Diagnosis and treatment forms vary from author to author. In 1979 the urological literature, unlike the dermatological literature, emphasized on the BXO urethral involvement.

In the 70's, Mallo et al. (16) and Herschorn et al. (17) showed histological findings of LS in the penile urethra. By contrast Khezri et al. (18) pursued that urethral damage was limited 
only to squamous meatus and the fossa navicularis without affecting the urethra. In 1998, Mundy et al. (19) published that 25\% of urethral stricture was due to LS. Barbagli et al. (20) in their 1999 work, described a percentage of 30\% with pathological diagnosis of LS, with involvement of the pan anterior urethra in 52\% and a higher incidence in patients with failed hypospadias. In a 2011 Barbagli et al. update (12), the authors found positive results in 85\% of biopsies, with bulbar urethra damage of $0 \%$. It is unclear why in some patients the complete anterior urethra is damaged showing a pan-urethritis radiological pattern (21) and why the bulbar or posterior urethra is not affected, which suggests a different epithelial origin as an explanation.

On the other hand, it has been thought that the urethral damage in patients with LS is not due to the disease itself, but because of the damage from continued expansion and infections. Venn et al. (19) surmised that changes to more proximal urethra may be due to chronic obstruction caused simply by emptying stenotic meatus, which leads to increased pressure and swelling of the posterior periurethral glands. It takes over 10 years for the disease to progress and affect the complete urethra. If the disease is limited to the skin it can be treated early with topic steroid treatment such as clobetasol propionate $0.05 \%$ or tacrolimus (22). Its purpose is to avoid the bother of the disease and prevent anatomical changes such as stenosis or malignant transformation, seen in $4-8 \%$ of cases $(2,23-29)$. Although some authors advocate the complete removal of the urethra to prevent the development of cancer, it may not be necessary as the urinary diversion would allow the drying of the distal urethra stopping the progression of LS and the cancer risk (30). Furthermore, patients with failed hypospadias show a high incidence of associated LS.

When the disease progresses despite medical treatment, we have the possibility of periodic urethral dilations, meatotomies, urethral removal or non-skin graft substitution, oral or tongue mucosa in sequential procedures and even definitive perineal urethrostomy. Heroic repair measures in one procedure are not usually justified due to the anticipated high failure rate $(6,9)$. Some patients decide after many previous procedures, not to have the second half of the procedure after a successful perineal urethrostomy (6). Other authors recommend using genital skin or bladder mucosa for repairing complete defects, but the low number of patients and limited follow-up prevent a conclusion being reached. The involvement of the perineostomy by LS has also been described, requiring subsequent surgical revision with low rates of success (15).

Intervening in early stages of the disease with a neo-meathotomy may involve stopping the spread of the disease. Peterson et al. reached success rates of 100\% for perineal urethrostomy in 52 patients (9), Kulkarni et al. report 72\% (15) arguing that the difference between the two series is due to the low number of previous urethroplasties in the first cohort, which could be considered a risk for subsequent strictures. Breyer et al. (21) stated that strictures greater than $4 \mathrm{~cm}$ or previous failed urethroplasty are predictive of failure. On the other hand, we observed a success rate of $100 \%$ in patients with no previous urethroplasty reaching significance in the univariate analysis. Similar to Barbagli's study (6), our major causes of stricture are the presence of LS and idiopathic (65\%). These authors achieved a success rate of $70 \%$ and defined as risk factors for failure, youth, prior urethroplasty and the presence of LS with no statistical study.

Our success rate reached $82.4 \%$. Although we could think a priori that the presence of radiation or surgery for penile carcinoma are factors for recurrence of stenosis, we did not find any statistical significance in our study probably due to the small number of these antecedents in the patient group. Radiation and ischemic scar are associated in many cases. In the same way erectile dysfunction is defined as an early symptom in the development of ischemic heart disease, we found that ischemic heart disease leads to poorer healing and greater failure of our perineostomies. If we eliminate patients with ischemic heart disease, we have a success rate of $100 \%$. For authors like Elhilali et al. (31), the incidence of urethral stricture is surprisingly high in patients with significant cardiac surgery. Urethral ischemia plays an important role in the development of stenosis. 
Therefore, the existence of the LS along with ischemic heart disease requires the use of non-skin terminal perineal urethrostomy techniques but those that maintain the highest degree of vascular flow in the urethral sponge as used in our group, similar to that reported by Myers et al. (10). We have acquired the experience in this technique from the two-stage procedures of Blandy et al. (7), which do not perform a complete section of the urethra, but a longitudinal open urethrotomy sutured to the skin edges, also carried out by Barbagli et al. (6). It is a misconception that in perineal urethrotomies of $2 \mathrm{~cm}$ it is necessary to prolong the incision to 4 or $6 \mathrm{~cm}$ as a prerequisite for success (10). Other authors propose the removal of the stenotic urethra, allowing greater mobility of the proximal urethra to suture the flap to the skin without stress, particularly in obese patients.

Our study has some limitations such as the small number of patients and its retrospective character. Although there are no questionnaires that assess patient satisfaction with the procedure and its finality, apparently it has been accepted by all patients, even more when it was not their first urethral surgery or they have severe limiting clinical problems in urination. In other series, younger patients had higher acceptance rates than patients older than 70. The acceptance of the procedure was shown by Barbagli et al. in their cohort (6). The perineostomy might not be accepted for religious, cultural, hygienic or psychological reasons, but, in fact, many patients already urinate while sitting when they reach this urethral situation (6, 9). Furthermore, the technique is fast and of little difficulty in its implementation and allows a quick return to normal physical activity, useful in older patients with comorbidity and multi-stepped and a fibrous urethral plate. Only those patients with damage or disease of the dorsal plate may receive a dorsal buccal mucosa urethral graft of adequate length to allow a suitable perineal urethrostomy and less retractable scar. The buccal mucosa is used in the subsequent repair of the perineal urethral stricture which continues to suffer from LS, and where $\mathrm{Y}-\mathrm{V}$ repair procedures are inadequate be- cause the disease affects the skin surrounding the perineal stoma.

\section{CONCLUSIONS}

Perineostomy is a valid alternative for patients with unfavorable urethral pathology (LS, failed previous urethroplasty, hypospadias repair history, prior pelvic radiation) or for individuals who do not to be submitted to more urethral or perineal urethroplasties.

Perineal urethrostomy success rate is affected principally by age, the presence of LS and ischemic vascular problems.

The preservation of the dorsal plate and longitudinal vascularization of the corpus spongiosum are advantageous factors to reduce healing complications after completion of the procedure.

\section{ABBREVIATIONS}

LS $=$ Lichen sclerosus

$\mathrm{BXO}=$ Balanitis xerotica obliterans

IHD $=$ Ischemic heart disease

\section{CONFLICT OF INTEREST}

None declared.

\section{REFERENCES}

1. Kulkarni S, Barbagli G, Sansalone S, Lazzeri M. One-sided anterior urethroplasty: a new dorsal onlay graft technique. BJU Int. 2009;104:1150-5.

2. Pugliese JM, Morey AF, Peterson AC. Lichen sclerosus: review of the literature and current recommendations for management. J Urol. 2007;178:2268-76.

3. Barbagli G, Selli C, Tosto A. Reoperative surgery for recurrent strictures of the penile and bulbous urethra. J Urol. 1996;156:76-7.

4. Barbagli G, De Angelis M, Palminteri E, Lazzeri M. Failed hypospadias repair presenting in adults. Eur Urol. 2006;49:887-94; discussion 895.

5. Elliott SP, McAninch JW, Chi T, Doyle SM, Master VA. Management of severe urethral complications of prostate cancer therapy. J Urol. 2006;176:2508-13. 
6. Barbagli G, De Angelis M, Romano G, Lazzeri M. Clinical outcome and quality of life assessment in patients treated with perineal urethrostomy for anterior urethral stricture disease. J Urol. 2009;182:548-57.

7. Blandy JP, Singh M, Tresidder GC. Urethroplasty by scrotal flap for long urethral strictures. Br J Urol. 1968;40:261-7.

8. Elliott SP, Eisenberg ML, McAninch JW. First-stage urethroplasty: utility in the modern era. Urology. 2008;71:889-92.

9. Peterson AC, Palminteri E, Lazzeri M, Guanzoni G, Barbagli G, Webster GD. Heroic measures may not always be justified in extensive urethral stricture due to lichen sclerosus (balanitis xerotica obliterans). Urology. 2004;64:565-8.

10. Myers JB, Porten SP, McAninch JW. The outcomes of perineal urethrostomy with preservation of the dorsal urethral plate and urethral blood supply. Urology. 2011;77:1223-7.

11. Johanson B. The reconstruction in stenosis of the male urethra. Z Urol. 1953;46:361-75.

12. Barbagli G, Mirri F, Gallucci M, Sansalone S, Romano G, Lazzeri M. Histological evidence of urethral involvement in male patients with genital lichen sclerosus: a preliminary report. J Urol. 2011;185:2171-6.

13. Barbagli G, Perovic S, Djinovic R, Sansalone S, Lazzeri M. Retrospective descriptive analysis of 1,176 patients with failed hypospadias repair. J Urol. 2010;183:207-11.

14. Perovic S, Barbagli G, Djinovic R, Sansalone S, Vallasciani $S$, Lazzeri M. Surgical challenge in patients who underwent failed hypospadias repair: is it time to change? Urol Int. 2010;85:427-35.

15. Kulkarni S, Barbagli G, Kirpekar D, Mirri F, Lazzeri M. Lichen sclerosus of the male genitalia and urethra: surgical options and results in a multicenter international experience with 215 patients. Eur Urol. 2009;55:945-54.

16. Mallo N, Garat JM, Santaularia J, Hernandez J. Urethrobalanitis xerotica obliterans. Eur Urol. 1978;4:9-12.

17. Herschorn S, Colapinto V. Balanitis xerotica obliterans involving anterior urethra. Urology. 1979;14:592-6.

18. Khezri AA, Dounis A, Dunn M. Balanitis xerotica obliterans. Br J Urol. 1979;51:229-31.

19. Venn SN, Mundy AR. Urethroplasty for balanitis xerotica obliterans. Br J Urol. 1998;81:735-7.

20. Barbagli G, Lazzeri M, Palminteri E, Turini D. Lichen sclerosis of male genitalia involving anterior urethra. Lancet. 1999;354:429.
21. Breyer BN, McAninch JW, Whitson JM, Eisenberg ML, Mehdizadeh JF, Myers JB, et al. Multivariate analysis of risk factors for long-term urethroplasty outcome. J Urol. 2010;183:613-7.

22. Hengge UR, Krause W, Hofmann H, Stadler R, Gross G, Meurer M, et al. Multicentre, phase II trial on the safety and efficacy of topical tacrolimus ointment for the treatment of lichen sclerosus. Br J Dermatol. 2006;155:1021-8.

23. Clouston D, Hall A, Lawrentschuk N. Penile lichen sclerosus (balanitis xerotica obliterans). BJU Int. 2011;108(Suppl 2):14-9.

24. Solsona E, Algaba F, Horenblas S, Pizzocaro G, Windahl T; European Association of Urology. EAU Guidelines on Penile Cancer. Eur Urol. 2004;46:1-8.

25. Paricio Rubio JF, Revenga AF, Alfaro TJ, Ramirez GT. Squamous cell carcinoma of the penis arising on lichen sclerosus et atrophicus. J Eur Acad Dermatol Venereol. 1999;12:153-6.

26. Powell J, Robson A, Cranston D, Wojnarowska F, Turner R. High incidence of lichen sclerosus in patients with squamous cell carcinoma of the penis. Br J Dermatol. 2001;145:85-9.

27. Pride HB, Miller OF 3rd, Tyler WB. Penile squamous cell carcinoma arising from balanitis xerotica obliterans. J Am Acad Dermatol. 1993;29:469-73.

28. Weber P, Rabinovitz H, Garland L. Verrucous carcinoma in penile lichen sclerosus et atrophicus. J Dermatol Surg Oncol. 1987;13:529-32.

29. Zaino RJ, Husseinzadeh N, Nahhas W, Mortel R. Epithelial alterations in proximity to invasive squamous carcinoma of the vulva. Int J Gynecol Pathol. 1982;1:173-84.

30. Depasquale I, Park AJ, Bracka A. The treatment of balanitis xerotica obliterans. BJU Int. 2000;86:459-65.

31. Elhilali MM, Hassouna M, Abdel-Hakim A, Teijeira J. Urethral stricture following cardiovascular surgery: role of urethral ischemia. J Urol. 1986;135:275-7.

Correspondence address: Juan Carlos Regueiro López, MD Avda Lagartijo, 22-3-1 Cordoba, 14005, Spain Telephone: + 3495 723-3131 E-mail: jcregueirolopez@gmail.com 


\section{EDITORIAL COMMENT}

Genital lichen sclerosus (LS) has been reported as a common progressive, sclerosing, inflammatory dermatosis disease. The incidence is $10.3 \%$ in a population of 2.096 patients (1). It is more prevalent in women than men (6:1-10:1). In women, this disease occurs, in general, in the menopause period, and in males at the age of 30-50 years. Surprisingly, it has also been reported in adolescents and children $(2,3)$.

In females, it affects the anogenital area (85-98\%) and extragenital area (15-20\%) (4). The differential diagnosis should include lichen planus, lichen simplex chronicus, vitiligo, alopecia, psoriasis and vulvar intraepithelial neoplasia (5). In males, this disease can affect, in the sequence, the foreskin and glans penis (57\%), urethral meatus (4\%), and can involve the anterior urethra (20\%) (4).

Urethral involvement by LS normally starts at the meatus and inches up to the anterior urethra until it reaches the bulb, involving the epithelial/spongiosus tissue displaying dilations and well demarcated stricture (panurethral stenosis, i.e., alternative multiple strictures in the anterior urethra). In the initial stage, degeneration of basal cell layer and inflammatory infiltration (lymphocytes, hyperkeratosis of the epithelium and stratum malpighii atrophy) have been verified (6).

The initial treatment, when the disease is limited to the foreskin/glands, consists in the application, topically or intralesionally, of steroids (clobetasol propionate, mometasone aceponate or betamethasone dipropionate cream or ointment) or emollients that might be used to inhibit chronic inflammatory processes $(4,7)$. As an alternative, pimecrolimus or tacrolimus can also be used (they have significant anti-inflammatory activity). Some benefits that are obtained, by reversing histological changes noted in the LS are known (2, 6). If the LS is confined to the foreskin, especially if the prepuce becomes tightened and nonretractile, circumcision is recommended. The possibility of cure by circumcision in the initial stages, i.e., when still located, is $92 \%(1,6,8)$, without recurrence signals. It is suggested that circumcision specimens always be sent for histological evaluation (to ensure diagnosis). As a strong association between LS and spongiosus cells carcinoma (SCC) has been reported (5), it is necessary to confirm the existence of LS and rule out SCC. It has been estimated that the risk of malignancy in male and female genitalia is $4-8 \%$ (9). For that reason, the treatment of panurethral strictures should never be managed with dilation or instrumentation.

Depasquale et al. noted that patients with LS limited to the glans penis and foreskin and who were submitted to circumcision alone did not show recurrent signals of LS at long-term surveillance (4). The perineal urethrostomy is commonly used as first step for staged reconstruction of the urethra $(10,11)$. With older patients or those with multiple failed repairs, with serious morbidity, or with histologically severe disease, the possibility of perineal urethrostomy might be discussed with the patient, especially elderly patients already accustomed to seat voiding. Moreover, failure in fibrotic hypospadias repair and postradiation stenosis in many situations requires a perineal urethrostomy. Because of many iterative interventions, many patients, by personal option, surprisingly have chosen this procedure as definitive, reporting satisfaction and acceptance (10).

In conclusion, to date the etiology of LS is not known. The pathology of this disease can lead to scarring causing serious alterations in the lower urinary tract function and also sexual dysfunction in males and females.

The diagnosis is eminently clinical, but it must be confirmed by histological evaluation. Treatment with corticosteroids has been effective enough. Surgical treatment is indicated when the foreskin, the coronal glans, the meatus or the urethra are involved.

\section{REFERENCES}

1. Bunker CB. Re: Sanjay Kulkarni, Guido Barbagli, Deepak Kirpekar, et al. Lichen sclerosus of the male genitalia and urethra: surgical options and results in a multicenter international experience with 215 patients. Eur Urol 2009;55:945-56.

2. Powell JJ, Wojnarowska F. Lichen sclerosus. Lancet. 1999;353:1777-83.

3. Das S, Tunuguntla HS. Balanitis xerotica obliterans--a review. World J Urol. 2000;18:382-7. 
4. Depasquale I, Park AJ, Bracka A. The treatment of balanitis xerotica obliterans. BJU Int. 2000;86:459-65.

5. Val I, Almeida G. An overview of lichen sclerosus. Clin Obstet Gynecol. 2005;48:808-17.

6. Stewart L, McCammon K, Metro M, Virasoro R. SIU/ICUD Consultation on Urethral Strictures: Anterior urethra-lichen sclerosus. Urology. 2014;83:S27-30.

7. Poynter JH, Levy J. Balanitis xerotica obliterans: effective treatment with topical and sublesional corticosteroids. $\mathrm{Br} \mathrm{J}$ Urol. 1967;39:420-5.

8. Morey AF, Lin HC, DeRosa CA, Griffith BC. Fossa navicularis reconstruction: impact of stricture length on outcomes and assessment of extended meatotomy (first stage Johanson) maneuver. J Urol. 2007;177:184-7; discussion 187.
9. Ranjan N, Singh SK. Malignant transformation of penile lichen sclerosus: exactly how common is it? Int J Dermatol. 2008;47:1308-9.

10. Barbagli G, De Angelis M, Romano G, Lazzeri M. Clinical outcome and quality of life assessment in patients treated with perineal urethrostomy for anterior urethral stricture disease. J Urol. 2009;182:548-57.

11. French D, Hudak SJ, Morey AF. The "7-flap" perineal urethrostomy. Urology. 2011;77:1487-9.

Jeová Nina Rocha, $M D, P h D$ Division of Urology, FMRP-USP Ribeirão Preto, SP, Brazil E-mail:jeova_rocha@yahoo.com 